The International Journal of Multimedia \& Its Applications (IJMA) Vol.6, No.5, October 2014

\title{
MINING IN ONTOLOGY WITH MULTI AGENT SYSTEM IN SEMANTIC WEB : A NOVEL APPROACH
}

\author{
Vishal Jain ${ }^{1}$ and Dr. S. V. A. V. Prasad ${ }^{2}$ \\ ${ }^{1}$ Research Scholar, Lingaya's University, Faridabad, Haryana, INDIA \\ ${ }^{2}$ Professor, Electronics and Communication Engineering Department, Lingaya's \\ University, Faridabad, Haryana, INDIA
}

\begin{abstract}
A large amount of data is present on the web. It contains huge number of web pages and to find suitable information from them is very cumbersome task. There is need to organize data in formal manner so that user can easily access and use them. To retrieve information from documents, there are many Information Retrieval (IR) techniques. Current IR techniques are not so advanced that they can be able to exploit semantic knowledge within documents and give precise results. IR technology is major factor responsible for handling annotations in Semantic Web (SW) languages. With the rate of growth of web and huge amount of information available on the web which may be in unstructured, semi structured or structured form, it has become increasingly difficult to identify the relevant pieces of information on the internet. IR technology is major factor responsible for handling annotations in Semantic Web (SW) languages. Knowledgeable representation languages are used for retrieving information. So, there is need to build an ontology that uses well defined methodology and process of developing ontology is called Ontology Development. Secondly, Cloud computing and data mining have become famous phenomena in the current application of information technology. With the changing trends and emerging of the new concept in the information technology sector, data mining and knowledge discovery have proved to be of significant importance. Data mining can be defined as the process of extracting data or information from a database which is not explicitly defined by the database and can be used to come up with generalized conclusions based on the trends obtained from the data. A database may be described as a collection of formerly structured data. Multi agents data mining may be defined as the use of various agents cooperatively interact with the environment to achieve a specified objective. Multi agents will always act on behalf of users and will coordinate, cooperate, negotiate and exchange data with each other. An agent would basically refer to a software agent, a robot or a human being Knowledge discovery can be defined as the process of critically searching large collections of data with the aim of coming up with patterns that can be used to make generalized conclusions. These patterns are sometimes referred to as knowledge about the data. Cloud computing can be defined as the delivery of computing services in which shared resources, information and software's are provided over a network, for example, the information super highway. Cloud computing is normally provided over a web based service which hosts all the resources required. As, the knowledge mining is used in many fields of study such as in science and medicine, finance, education, manufacturing and commerce. In this paper, the Semantic Web addresses the first part of this challenge by trying to make the data also machine understandable in the form of Ontology, while Multi-Agent addresses the second part by semi-automatically extracting the useful knowledge hidden in these data, and making it available.
\end{abstract}

DOI : 10.5121/ijma.2014.6504 
The International Journal of Multimedia \& Its Applications (IJMA) Vol.6, No.5, October 2014

\section{KEYWORDS}

Information Retrieval, Semantic Web, Ontology, Ontology Mapping, Indexing with Ontology, JADE, MultiAgent Systems.

\section{INTRODUCTION}

A large amount of data is present on the web. It contains huge number of web pages and to find suitable information from them is very cumbersome task. There is need to organize data in formal manner so that user can easily access and use them. To retrieve information from documents, there are many Information Retrieval (IR) techniques. Current IR techniques are not so advanced that they can be able to exploit semantic knowledge within documents and give precise results. IR technology is major factor responsible for handling annotations in Semantic Web (SW) languages [2]. With the rate of growth of web and huge amount of information available on the web which may be in unstructured, semi structured or structured form, it has become increasingly difficult to identify the relevant pieces of information on the internet. The need is to organize this data in a formal system which results in more relevant end useful and structured information. So, it has become necessary for users to utilize automated tools in finding the desired information resources, and to track and analyze their navigation patterns. In this context, the role of user modelling and personalized information access is increasing. Ontology may be a mechanism for obtaining the information on web in a more structured way through Semantic Web. This work focuses on the problem of choosing a representation of documents that can be suitable to induce concept-based user profiles as well as to support a content-based retrieval process. It is at this juncture that the science of Agent Mining comes to rescue. The Semantic Web addresses the first part of this challenge by trying to make the data also machine understandable in the form of Ontology, while Multi-Agent addresses the second part by semi-automatically extracting the useful knowledge hidden in these data, and making it available. As current web 2.0 is shifting towards web 3.0, there is need to upgrade technologies according to the same.

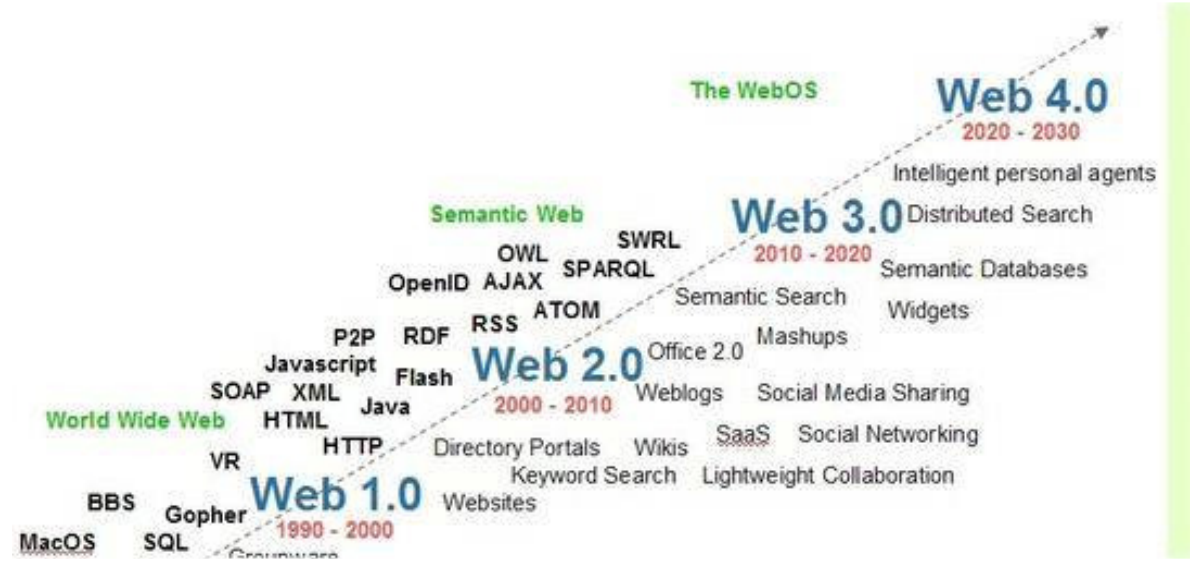

Figure 1: Current Web to Semantic Web 
The International Journal of Multimedia \& Its Applications (IJMA) Vol.6, No.5, October 2014

\section{LITERATURE SURVEY}

Anarosa Alves, Franco Brandão, Viviane Torres da Silva, Carlos José Pereira de Lucena [10], presented a work on a model driven approach to develop multi-Agent Systems. In this paper, authors described a model driven approach to develop multi-agent systems that begins with an ontology based on the TAO conceptual framework.

Quynh-Nhu Numi Tran, Graham Low [11], worked on MOBMAS: A methodology for ontologybased multi-agent systems development. In this authors, authors proposed a new framework and compared MOBMAS against sixteen well known methodologies: MaSE, MASSIVE, SODA, GAIA, MESSAGE , Methodology for BDI Agent, INGENIAS, Methodology with High-Level and Intermediate Levels, Methodology for Enterprise Integration, PROMETHEUS, PASSI, ADELFE, COMOMAS , MAS-Common, KADS , CASSIOPEIA and TROPOS.

Pakornpong Pothipruk and Pattarachai Lalitrojwong [12], worked on an Ontology-based Multiagent System for Matchmaking. Csongor Nyulas, Martin J. O'Connor, Samson Tu1, David L. Buckeridge, Anna Akhmatovskaia, Mark A. Musen [13], presented their work on An OntologyDriven Framework for Deploying JADE Agent Systems. Authors described a methodology and suite of tools to support the modeling and deployment of agents on the JADE platform. These models are encoded using the Semantic Web ontology language OWL and provide detailed computer-interpretable specifications of agent behavior in a JADE system.

Gajun Ganendran, Quynh-Nhu Tran, Pronab Ganguly, Pradeep Ray and Graham Low [14], proposed a methodology on An Ontology-driven Multi-agent approach for Healthcare. In this paper, authors described an ontology-driven multi-agent approach to the development of healthcare systems, with a case study in diabetes management.

Wongthongtham, P., Chang, E., Dillon, T.S. [15], worked on Ontology-based Multi-agent system to Multi-site Software Development. Authors described software agent utilized ontology as its intelligence in MSSD and found that it has benefits. Ontology gives computers more knowledge that the agent can utilize

Maja Hadzic, Elizabeth Chang [16], worked on use of ontology-based multi-agent systems in the biomedical domain. Authors have shown how the ontologies can be used by multi-agent systems in intelligent information retrieval processes. The ontologies can be used to support some important processes involved in the information retrieval such as posing queries by the user, problem decomposition and task sharing among different agents, result sharing and analysis, information selection and integration, and structured presentation of the assembled information to the user.

\section{Problem Definition}

Internet is considered as repository of collection of web documents. There is plethora of documents available on web are very difficult to access and extract relevant information from them. The reason is uncertainty of huge documents that confuse users by providing several keywords and results in context of given query. The documents may be unstructured, structured and semi-structured. It is known that mostly of these documents are unstructured. Although there is larger number of documents residing on web storage house, still the users are unable to find 
The International Journal of Multimedia \& Its Applications (IJMA) Vol.6, No.5, October 2014

relevant information about given domain. The reason is the uncertainty of documents that creates users in dilemma by providing hundreds of results and keywords in response to given query. When a user wants to find some information, he/she enters a query and results are produced via hyperlinks linked to various documents available on web. But the information that is retrieved to us may or may not be relevant. This irrelevance is caused due to huge collection of documents available on web. Traditional search engines are based on keyword based searching that is unable to transform raw data into knowledgeable representation data. It is a cumbersome task to extract relevant information from large collection of web documents. These shortcomings have led to the concept of Semantic Web and Ontology into existence.

\section{INFORMATION RETRIEVAL}

Information Retrieval involves identifying and extracting relevant pages containing that specific information according to predefined guidelines. There are many IR techniques for extracting keywords like NLP based extraction techniques which are used to search for simple keywords. Then we have Aero Text system for text extraction of key phrases from text documents. Information Retrieval, retrieve only structured data. But IR provides all types of information access such as analysis, organization, storage, searching, retrieval of information and structured data. As according to Slaton's classic textbook: "Information retrieval is a field concerned with the structured, analysis, organization, storage, searching, and retrieval of information". Information retrieval is defined by Carlo Meghini et al. "Information Retrieval as the task of identifying documents in a collection on the basis of properties described to the documents by the user requesting the retrieval" . Information retrieval is a process of retrieval-structured data from the unstructured data.

\section{SEMANTIC WEB}

The idea of Semantic Web (SW) as envisioned by Tim Bermers Lee came into existence in 1996 with the aim to translate given information into machine understandable form [7]. The Semantic Web (SW) is an extension of current www in which documents are filled by annotations in machine understandable markup language. It is defined as framework of expressing information to develop various languages and approaches for increasing IR effectiveness. Semantic Web uses documents called Semantic Web Documents (SWD's) that are written in SW languages like OWL, DAML+OIL. SW is an XML (Extensible Markup Language) application. Its aim is to maintain coordination between users and software agents so that they can find answers to their queries clearly.

\section{ONTOLOGY}

The term ontology can be defined in many different ways. Genersereth and Nilsson defined Ontology as an explicit specification of a set of objects, concepts, and other entities that are presumed to exist in some area of interest and the relationships that hold them [8]. Ontology defines a common vocabulary for researchers who need to share common information in a domain". It enables the Web for software components can be ideally supported through the use of Semantic Web technologies. This helps in understanding the concepts of the domain as well as helps the machine to interpret the definitions of concepts in the domains and also the relations between them. 
The International Journal of Multimedia \& Its Applications (IJMA) Vol.6, No.5, October 2014

Ontologies can be broadly divided into two main types: lightweight and heavyweight. Lightweight Ontologies involve taxonomy (or class hierarchy) that contains classes, subclasses, attributes and values. Heavy weight Ontologies model domains in a deeper way and include axioms and constraints. Ontology layer consists of hierarchical distribution of important concepts in the domain and describing about the Ontology concepts, relationships and constraints. Figure 1.9 displays the Ontology and its Constituents parts.

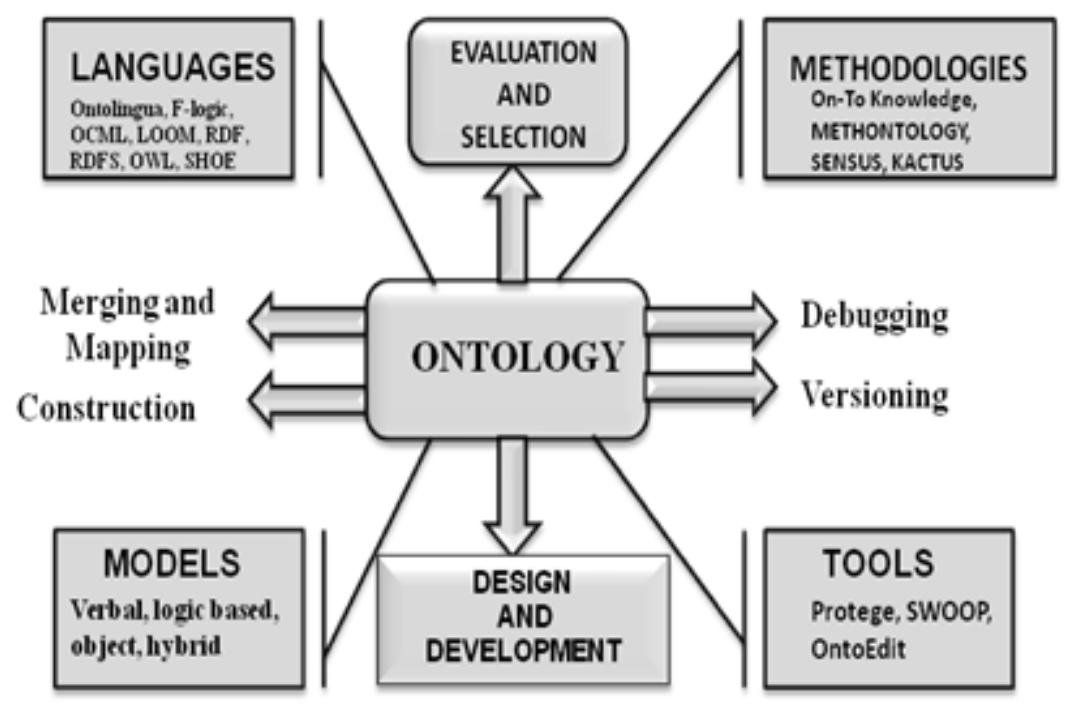

Figure 1: "Ontology and its Constituents [3]"

\section{Multi Agent SySTEM}

Multi-agent systems have been successful in the distributed implementation of KM processes. Knowledge acquisition agents have been one of the most successful applications of software agents, specifically in the Internet, where knowledge-collector agents operate within available information resources and validate them in accordance with the users' interests. On the other hand, knowledge transfer lies in an end-to-end routing of knowledge that is generated by some actor, and it is another typical task that has been realized by software agents. Therefore, it is reasonable to approach the multi-agent paradigm for knowledge production. Knowledgeproducing agents need to do formulations in keeping with a validation scheme that supports the knowledge construction.

Multi-agent systems can support the coordinated interaction needed to achieve an agreement on the knowledge that is eventually generated. They can also support the validation scheme. Advances in multi-agent systems as an alternative way to build distributed systems have made agents a facilitator of human-computer interaction. Agents have been proven as helpful tools for the coordination of people who are performing a given task. Agent interaction protocols govern the exchange of a series of messages among agents, in accordance with the interaction style among actors.

The interaction styles of individual agents can be competitive, cooperative or negotiating. But, both the group behavior and the authoring of knowledge objects are social, group-level issues, more than individual subjects, and it is there where multi-agent systems can give their 
The International Journal of Multimedia \& Its Applications (IJMA) Vol.6, No.5, October 2014

contribution. The architecture presented in this work is a bottom-up, multi-agent approach for knowledge production. Our working hypothesis is that a group of agents can help in the collaborative production of knowledge by coordinating their creation activities. Therefore, different agents can act as representatives of knowledge-producing actors, according to the following principles:

- Agents can be structured into separable knowledge domains of interaction. This structuring reflects the knowledge differences between developers.

- A dynamic rethinking of the structure of interactions in different domains can help to reduce conflicts during.

\section{PRoposed SOLUTiON}

Semantic Web is a well defined portal that helps in extracting relevant information using many Information Retrieval techniques. Current Information Retrieval techniques are not so advanced that they can be able to exploit semantic knowledge within documents and give precise result. The terms, Information Retrieval, Semantic Web and Ontology are used differently but they are interconnected with each other. Information Retrieval technology and Web based Indexing contributes to existence of Semantic Web. Use of Ontology also contributes in building new generation of web- Semantic Web. With the help of ontologies, make content of web as it will be markup with the help of Semantic Web documents can be possible. Ontology is considered as backbone of Software system. It improves understanding between concepts used in Semantic Web. So, there is need to build an ontology that uses well defined methodology and process of developing ontology is called Ontology Development. Ontology can be used to build structured data and Multi-Agent system for information extraction from the ontology database. In order to realize with Multi Agent Systems and interact with their agents, a framework is developed that is called JADE (Java Agent Development Environment). It is considered as middleware that implies agent platform and development framework.

The study of the related works and literature review that in order to retrieve meaningful information from the data warehouse through the help of a multi-agent system and data mining techniques in a cloud computing environment, the following architecture is designed [20]:The Infrastructure-as-a-Service (IaaS) provides a virtual environment with storage and network without having physical hardware. Therefore, Infrastructure cloud computing provides a data warehouse for storage of data for further analysis. The user will submit a flat-text based request on the IaaS for information retrieval from its integrated data warehouse that has gathered data from numerous areas of business to present to the user a wide variety to choose from. The IaaS will forward this request to the MAS to find the information as requested. However, MAS does not have the ability to find the information that has large amounts of data from a data warehouse. Therefore, it will use the data mining algorithm to analyze the large amount of data from the data warehouse that is residing in the IaaS.As a pre-processing stage, the MAS will first develop a target data set which will be large enough to contain all the possible data patterns and send it into he system. Then the processing will begin where the data will be analyzed through anomaly detection, clustering, classification, regression and summarization. The result of the process will be shown on the screen to the user. 


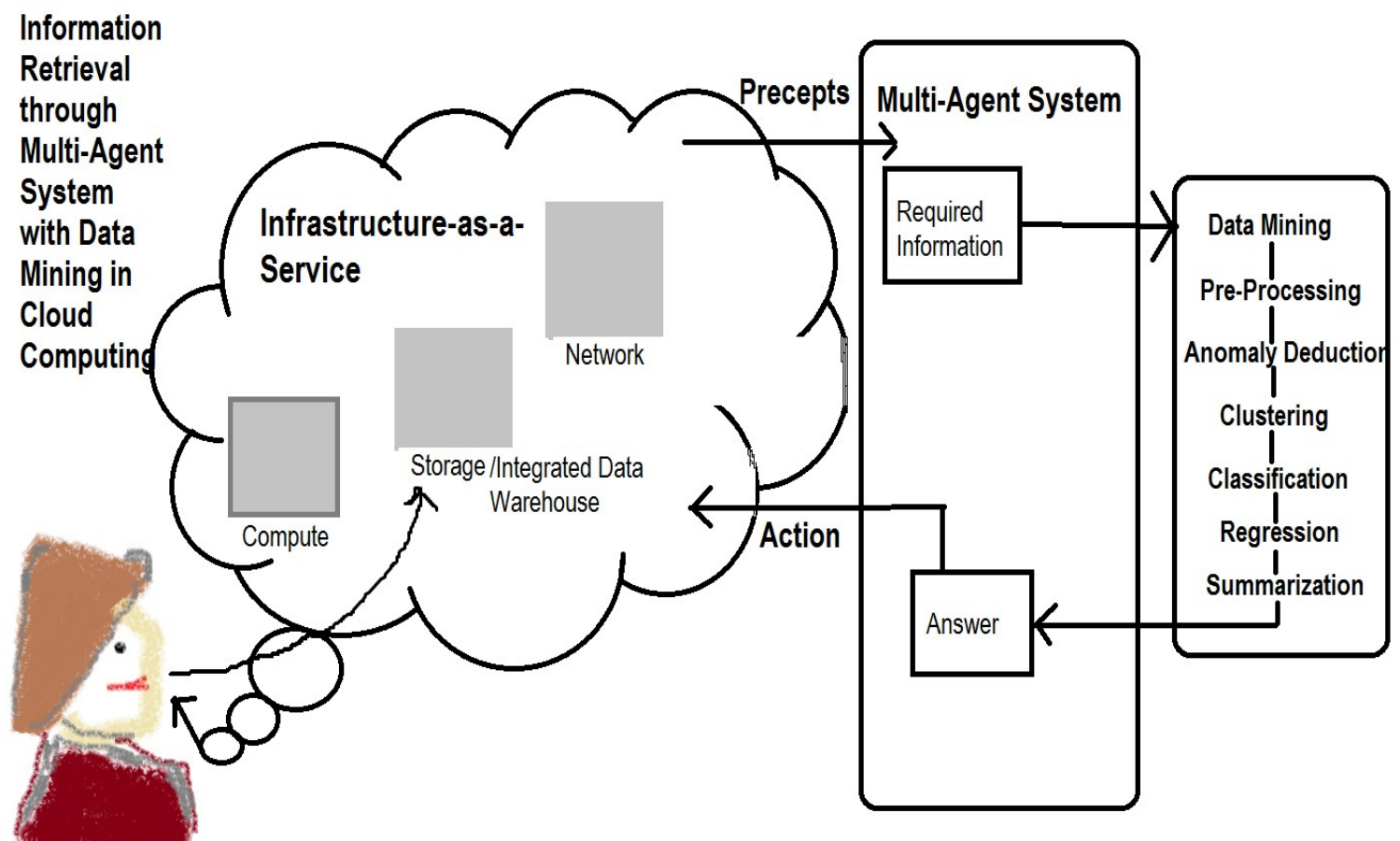

Figure 2: "Information Retrieval through Multi-Agent System with Data Mining in Cloud Computing" [20]

\section{TECHNOLOGY STACK}

1. OWL: Web ontology languageis used here to represent the university domain including, Universities, Courses, People, Library and Materials.

2. Protégé: A free, open-source ontology editor and framework for building intelligent systems

3. OWLIM: OWLIM is a family of semantic repositories, or RDF database management systems, with the following characteristics:

a. native RDF engines, implemented in Java

b. delivering full performance through both Sesame and Jena

c. robust support for the semantics of RDFS, OWL 2 RL and OWL 2 QL

d. best scalability, loading and query evaluation performance

4. WEKA - Apriori Algorithm: Weka is a collection of machine learning algorithms for solving real-world data mining problems. It is written in Java and runs on almost any platform. The Apriori Algorithm is an influential algorithm for mining frequent itemsets for boolean association rules.

5. Empire: Empire provides Java developers an easy way to integrate and start using SPARQL \& RDF via JPA persistence annotations.

6. JADE: Java Agent DEvelopment Framework, or JADE, is a software for the development of agents, implemented in Java. JADE system supports coordination between several agents FIPA and provides a standard implementation of the communication language FIPA-ACL, which facilitates the communication between agents and allows the services detection of the system 


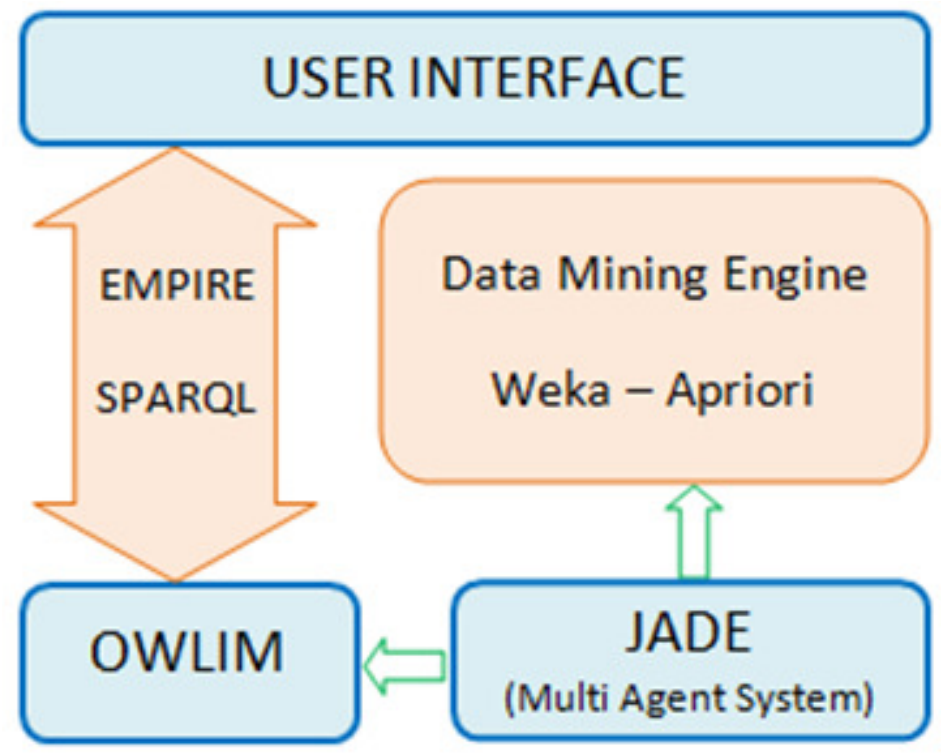

Figure 3: "Propose Framework"

\section{CONCLUSION}

The information retrieval practical model through the multi-agent system with data mining in a cloud computing environment has been proposed. It is however, recommended that users should ensure that the request made to the IaaS is within the scope of integrated data warehouse and is clear and simple. Thus, making the work for the multi-agent system easier through application of the data mining algorithms to retrieve meaningful information from the data warehouse. In this proposed research model/architecture, the use of cloud computing allows the users to retrieve meaningful information from virtually integrated data warehouse that reduces the costs of infrastructure and storage. In this paper, new proposed model has been proposed; "Mining in Ontology with Multi Agent Systems" has been discussed. In this model, the Semantic Web addresses the first part of this challenge by trying to make the data also machine understandable in the form of Ontology, while Multi-Agent addresses the second part by semi-automatically extracting the useful knowledge hidden in these data, and making it available. This model can be developed, which may give better results as compare to RDBMS. This paper will also helpful to other researchers, who would like to do work in this area.

\section{ACKNOWLEDGEMENTS}

I, Vishal Jain, would like to give my thanks to Dr. M. N. Hoda, Director, Bharati Vidyapeeth's Institute of Computer Applications and Management (BVICAM), New Delhi, for giving me opportunity to do Ph.D from Linagay's University, Faridabad, Haryana.

\section{REFERENCES}

[1] Vishal Jain, Gagandeep Singh, Dr. Mayank Singh, "Ontology Development Using Hozo and Semantic Analysis for Information Retrieval in Semantic Web", 2013 IEEE Second International Conference on Image Information Processing (ICIIP -2013) held on December 9 - 11, 2013, organized 
The International Journal of Multimedia \& Its Applications (IJMA) Vol.6, No.5, October 2014

by Jaypee University of Information Technology, Waknaghat, Shimla, Himachal Pradesh, INDIA and proceeding published by the IEEE.(Available at:

http://ieeexplore.ieee.org/xpl/login.jsp?tp=\&arnumber=6707566\&url=http\%3A\%2F\%2Fieexplore.ie ee.org\%2Fxpls\%2Fabs_all.jsp\%3Farnumber\%3D6707566)

[2] Gagandeep Singh, Vishal Jain, "Information Retrieval through Semantic Web: An Overview", Confluence 2012, held on 27th and 28th September, 2012 page no.114-118, at Amity School of Engineering \& Technology, Amity University, Noida.

[3] Vishal Jain, Dr. Mayank Singh, "Ontology Development and Query Retrieval using Protégé Tool”, International Journal of Intelligent Systems and Applications (IJISA), Hongkong, Vol. 5, No. 9, August 2013, page no. 67-75, having ISSN No. 2074-9058, DOI: 10.5815/ijisa.2013.09.08 and index with Thomson Reuters (Web of Science), EBSCO, Proquest, DOAJ, Index Copernicus.(Available at: http://www.mecs-press.org/ijisa/ijisa-v5-n9/IJISA-V5-N9-8.pdf)

[4] Vishal Jain, Dr. Mayank Singh, "Ontology Based Information Retrieval in Semantic Web: A Survey", International Journal of Information Technology and Computer Science (IJITCS), Hongkong, Vol. 5, No. 10, September 2013, page no. 62-69, having ISSN No. 2074-9015, DOI: 10.5815/ijitcs.2013.10.06 and index with Thomson Reuters (Web of Science), EBSCO, Proquest, DOAJ, Index Copernicus

[5] Anarosa Alves, Franco Brandão, Viviane Torres da Silva, Carlos José Pereira de Lucena , "A Model Driven Approach to Develop Multi-Agent Systems”, Monografias em Ciência da Computação, No. 09/05.

[6] Quynh-Nhu Numi Tran, Graham Low, "MOBMAS: A Methodology for Ontology-Based MultiAgent Systems Development”, Information and Software Technology 50 (2008) 697-722.

[7] Pakornpong Pothipruk and Pattarachai Lalitrojwong, "An Ontology-based Multi-agent System for Matchmaking"

[8] Csongor Nyulas, Martin J. O’Connor, Samson Tu1, David L. Buckeridge, Anna Akhmatovskaia, Mark A. Musen, “An Ontology-Driven Framework for Deploying JADE Agent Systems”, Stanford University, Stanford Center for Biomedical Informatics Research.

[9] Gajun Ganendran, Quynh-Nhu Tran, Pronab Ganguly, Pradeep Ray and Graham Low, “An Ontologydriven Multi-agent approach for Healthcare”, HIC 2002, 0958537097.

[10] Wongthongtham, P., Chang, E., Dillon, T.S., "Ontology-based Multi-agent system to Multi-site Software Development”, Workshop QUTE-SWAP@ACM/SIGSOFT-FSE12, November 5, 2004.

[11] Maja Hadzic, Elizabeth Chang, "Use Of Ontology-Based Multi-Agent Systems in the Biomedical Domain", Curtin University of Technology, School of Information Systems Perth, Western Australia, 6845, Australia.

[12] Vishal Jain, Dr. Mayank Singh, “Architecture Model for Communication between Multi Agent Systems with Ontology", International Journal of Advanced Research in Computer Science (IJARCS), Volume.4 No.8, May-June 2013, page no. 86-91 with ISSN No. 0976 - 5697 and indexed with EBSCO HOST Index Copernicus, DOAJ, ICV value is 5.47.(Available at: http://ijarcs.info/?wicket:interface=:2::::)

[13] Gagandeep Singh, Vishal Jain, Dr. Mayank Singh, “ An Approach For Information Extraction using Jade: A Case Study”, Journal of Global Research in Computer Science (JGRCS), Vol.4 No. 4 April, 2013, page no. 186-191, having ISSN No. 2229-371X , with impact factor (2012) 0.60.(Available at: http://jgrcs.info/index.php/jgrcs/article/view/640/501)

[14] Vishal Jain, Gagandeep Singh, Dr. Mayank Singh, "Implementation of Multi Agent Systems with Ontology in Data Mining", International Journal of Research in Computer Application and Management (IJRCM) May, 2013 page no. 108-114 having ISSN No. 2231 - 1009 and index with Index Copernicus, DOAJ, J-Gate, Ulrich's, EBSCO, Poland IC Value is 5.09. (Available at: http://ijrcm.org.in/comapp/index.php?type=Archives)

[15] Bouchiha Djelloul, Malki Mimoun, and Mostefai Abd El Kader, "Towards Reengineering Web Applications to Web Services", The International Arab Journal of Information Technology (IAJIT), Vol. 6, No. 4, October 2009 
The International Journal of Multimedia \& Its Applications (IJMA) Vol.6, No.5, October 2014

[16] Sidi Benslimane, Mimoun Malki, and Djelloul Bouchiha, "Deriving Conceptual Schema from Domain Ontology: A Web Application Reverse Engineering Approach", The International Arab Journal of Information Technology (IAJIT), Vol. 7, No. 2, April 2010

[17] Accessible from Mr.Lobo L.M.R.J, Sunita B Aher, "Data Mining in Educational System using WEKA", "International Conference on Emerging Technology Trends (ICETT)", 2001.

[18] Accessible from Sonali Agarwal, Neera Singh, Dr. G.N. Pandey, "Implementation of Data Mining and Data Warehouse in E-Governance", "International Journal of Computer Applications (IJCA) (0975-8887), Vol.9-No.4,” November 2010.

[19] Fensel, Dieter. "Ontologies: A Silver Bullet for Knowledge Management and Electronic Commerce". Heidelberg: Springer, 2003.

[20] Vishal Jain, Mahesh Kumar Madan, "Information Retrieval through Multi-Agent System with Data Mining in Cloud Computing", International Journal of Computer Technology and Applications (IJCTA) Volume 3 Issue 1, January-February 2012, page no. 62-66.

[21] N Sivaram, K.Ramar, "Applicability of Clustering and Classification Algorithms for Recruitment Data Mining", "International Journal of Computer Applications (IJCA), Vol.4, No-5", March 2010.

[22] Ferenc Bodon, "A fast Apriori implementation", "In Proceedings of the IEEE ICDM Workshop on Frequent Item set Mining Implementations", 2003.

[23] Tan Pang-Ning, "An Introduction to Data Mining". "Pearson Education", 2007.

[24] U.K. Pandey, and S.Pal, "Data Mining: A Prediction of performer using Classification", "International Journal of Computer Science and Information Technology (IJCSIT), Vol.2(2), IISN:0975-9646”, pages 686-690, 2011.

[25] K Saravana Kumar, R. Manicka Chezian, "A Survey on Association Rule Mining using Apriori Algorithm", "International Journal of Computer Applications (IJCA), Vol.45-Number 5", 2012.

[26] Amirmahadi Mohammadighavam, Neda Rajabpour, Ali Naserasadi, "A Survey on Data Mining Approaches", "International Journal of Computer Applications (IJCA) (0975-8887) Vol.36-Number 6", 2011.

[27] Dhanashree S. Deshpande, "A Survey on Web Data Mining Applications", "International Journal of Computer Applications (IJCA), ETCSIT- Number 3", 2012.

[28] Antonopoulos, Nick and Lee Gillam. "Cloud Computing: Principles, Systems and Applications". New York: Springer, 2010.

\section{AUTHORS}

Vishal Jain has completed his M.Tech (CSE) from USIT, Guru Gobind Singh Indraprastha University, Delhi and doing P.hD from Computer Science and Engineering Department, Lingaya's University, Faridabad. Presently he is working as Assistant Professor in Bharati Vidyapeeth's Institute of Computer Applications and Management, (BVICAM), New Delhi. His research area includes Web Technology, Semantic Web and Information Retrieval. He is also associated with CSI, ISTE.

Dr. S. V. A. V. Prasad has completed M.Tech., Ph.D. Presently working as professor and Dean (Academic Affairs), Dean (R\&D, IC), Dean School of Electrical Sciences. Sir has actively participated and organized many refresher courses, seminars, workshops on ISO, ROHS, Component Technology, WEEE, Organizational Methods, Time Study, Productivity enhancement and product feasibility etc. Sir has developed various products like $15 \mathrm{MHz}$ dual Oscilloscope, High Voltage Tester, VHF Wattmeter, Standard Signal,

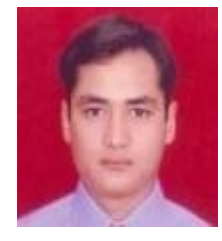
Generator with AM/FM Modulator, Wireless Becom, High power audio Amplifier, Wireless microphone and many more in the span of 25 years $(1981-2007)$. Sir has awarded for excellence in R\&D in year 1999, 2004 and National Quality Award during the Year 1999, 2000, 2004 and 2006.Sir is Fellow member of IEEE and life member of ISTE, IETE, and Society of Audio \& Video System. Sir has published more than 90 research papers in various National \& International conferences and journals. Sir research area includes Wireless Communication, Satellite Communication \& Acoustic, Antenna, Neural Networks, and Artificial Intelligence. 